$R M x A C, \mathbf{5 3}, 83-90(2021)$

(c) 2021: Instituto de Astronomía, Universidad Nacional Autónoma de México

https://doi.org/10.22201/ia.14052059p.2021.53.19

\title{
OPTICAL SPECTROSCOPY OF CANDIDATES IN THE LIGO/VIRGO BINARY MERGER ERROR BOXES
}

\author{
A. F. Valeev ${ }^{1}$, A. J. Castro-Tirado ${ }^{2,3}$, Y. -D. Hu², V. V. Sokolov ${ }^{1}$, I. Agudo ${ }^{2}$, M. D. Caballero-García ${ }^{4}$, \\ J. Cepa ${ }^{5}$, J. A. Font ${ }^{6,15}$, S. Jeong ${ }^{7}$, A. Martin-Carrillo ${ }^{8}$, S. B. Pandey ${ }^{9}$, E. Pian ${ }^{10}$, S. R. Oates ${ }^{11}$, \\ R. Sánchez-Ramírez ${ }^{12}$, A. M. Sintes ${ }^{13}$, B.-B. Zhang ${ }^{14}$, and E. Fernandez-Garcia ${ }^{2}$
}

\section{RESUMEN}

Hemos realizado observaciones espectrocópicas de candidatos hallados en zonas de error de ondas gravitacionales (S190408an, S190425z, S190426c, S190510g, S190728q, S190814bv). Presentamos la clasificación espectral de 34 fuentes transitorias realizadas con el Gran Telescopio Canarias de $10.4 \mathrm{~m}$ para las alertas reportadas con anterioridad al 1 de septiembre de 2019. Descartamos su relación con las alertas gravitacinales.

\section{ABSTRACT}

We performed optical spectroscopy of the candidates inside the gravitational wave errorboxes ( S190408an, S190425z, S190426c, S190510g, S190728q, S190814bv). The spectral classification of 34 transients observed with the $10.4 \mathrm{~m}$ Gran Telescopio de Canarias prior to 1 Sep 2019 is presented. We ruled out the association of these candidates with gravitational wave events.

Key Words: gravitational waves — methods: spectral classification — methods: spectroscopic redshift — stars: black holes — stars: neutron

\section{INTRODUCTION}

When compact binary star systems merge, they release large amounts of energy in the form of gravitational waves (Abbott \& LIGO \& Virgo Scientific Collaboration 2017, 2016). If the system is either a neutron star and stellar mass black hole (NSBH) or

\footnotetext{
${ }^{1}$ Special Astrophysical Observatory, Nizhnij Arkhyz 369167, Russia (azamat@sao.ru).

${ }^{2}$ Instituto de Astrofísica de Andalucia (IAA-CSIC), Glorieta de la Astronomia sn, E-18008, Granada, Spain.

${ }^{3}$ Unidad Asociada Departamento de Ingeniería de Sistemas y Automática, E.T.S. de Ingenieros Industriales, Universidad de Málaga, Spain.

${ }^{4}$ Astronomical Institute of the Academy of Sciences, Boční II 1401, CZ-14100 Praha 4, Czech Republic.

${ }^{5}$ Instituto de Astrofísica de Canarias, Vía Láctea s/n, 38205 Santa Cruz de La Laguna, Tenerife, Spain.

${ }^{6}$ Departamento de Astronomía y Astrofísica, Universitat de València, Dr. Moliner 50, 46100, Burjassot (València), Spain.

${ }^{7}$ Institute for Science and Technology in Space, SungKyunKwan University, Suwon 16419, Korea.

${ }^{8}$ University College Dublin, Ireland.

${ }^{9}$ Aryabhatta Research Institute of Observational Sciences, Manora Peak, Nainital 263 002, India.

${ }^{10}$ INAF, Astrophysics and Space Science Observatory, via P. Gobetti 101, 40129 Bologna, Italy.

${ }^{11}$ University of Birmingham, United Kingdom.

${ }^{12}$ INAF, Istituto di Astrofisica e Planetologia Spaziali, Rome, Italy.

${ }^{13}$ Universitat de les Illes Balears, Spain.

${ }^{14}$ School of Astronomy and Space Science, Nanjing University, 210093, Nanjing, China.

${ }^{15}$ Observatori Astronòmic, Universitat de València, C/ Catedrático José Beltrán 2, 46980, Paterna (València), Spain.
}

a binary neutron star (BNS), the merger is expected to be accompanied by various electromagnetic phenomena.

The discovery of GW170817 by LIGO and Virgo and its electromagnetic counterparts (shortduration GRB 170817A, detected by Fermi/GBM and INTEGRAL/SPI-ACS, optical AT2017gfo, investigated in optical and infrared wavelenghts, etc.) opened a new era in the Multi-Messenger Astronomy (Abbott 2017).

The firstly discovered electromagnetic counterpart of the gravitational waves from a BNS merger required the developing of an optimal search strategy. The discovery of GW170817 counterpart using a galaxy-targeted strategy (Gehrels et al. 2016) means that this method can be very effective. However, it requires a large catalog of galaxies with photometric redshifts and a way to measure its completeness to distances relevant for advanced LIGO and Virgo.

Whatever method is chosen to search for electromagnetic counterparts, the definitive classification of transients requires the spectral observations. The most straightforward way of ruling out association of a GW event with an electromagnetic transient is to prove that the latter occurred before the GW signal or the redshift of its host galaxy is inconsistent with the GW distance inferred from the gravitational signal under standard sirene assumption. The spectral classification of a transient may identify a source as 
a different kind of transient event with well known progenitors (like $\mathrm{SN}$ ).

In the next section we present the spectral classification of the discovered transients in six errorboxes of the GW event candidates announced during the O3 run of advanced LIGO and Virgo (S190408an, S190425z, S190426c, S190510g, S190728q, S190814bv). We limit our target list to the candidates observed prior 2019 Sep, 1. Our spectral classification is summarized at Tab. 1

\section{OBSERVATIONS AND CLASSIFICATION}

We observed potential candidates in GW localisation errorboxes with the 10.4-m Gran Telescopio de Canarias (GTC) (PI: A. Castro-Tirado), located at the observatory of Roque de los Muchachos in La Palma (Canary Islands, Spain), equipped with the Optical System for Imaging and low-intermediateResolution Integrated Spectroscopy (OSIRIS) (Cepa et al. 2000).

Optical images were usually taken for the candidates in the r-band filter. The deep photometry (griz filters) was performed in case of faint sources. We performed PSF photometry of the target. Photometric zero points were computed using the PanSTARRS catalogue stars as secondary standards.

GTC/OSIRIS spectra for the targets were obtained either with the R1000B or with the R1000R/R2500I grisms and a 1 arcsec slit covering the $3,700-7,500 \AA$ or $5,100-10,000 \AA$ range. The slit was placed in order to cover the candidate location and the host galaxy center. Data were reduced and calibrated using standard routines.

Quadrants, which were made from GAIA DR2 stars, were used to calculate the astrometric solution applying solve-field routine from astrometry.net package. Using $\mathrm{iraf} /$ apall and $\mathrm{iraf} /$ phot packages we extracted the 1-D spectrum of transient and performed aperture photometry. We have classified the transient using the supernova template matching code SNID (Blondin \& Tonry 2007).

\section{S190408AN}

The S190408an (Ligo Scientific Collaboration \& VIRGO Collaboration 2019a) event was detected by three detectors (LIGO Hanford Observatory (H1), LIGO Livingston Observatory (L1), and Virgo Observatory (V1)) at 2019-04-08 18:18:02 UTC. The four pipelines showed the binary black holes with high propability (>99\%). The distance estimation is $\mathrm{d}=1473 \pm 358 \mathrm{Mpc}$ and the error box $82 / 387 \mathrm{deg}^{2}$ for $50 / 90 \%$ confidence.

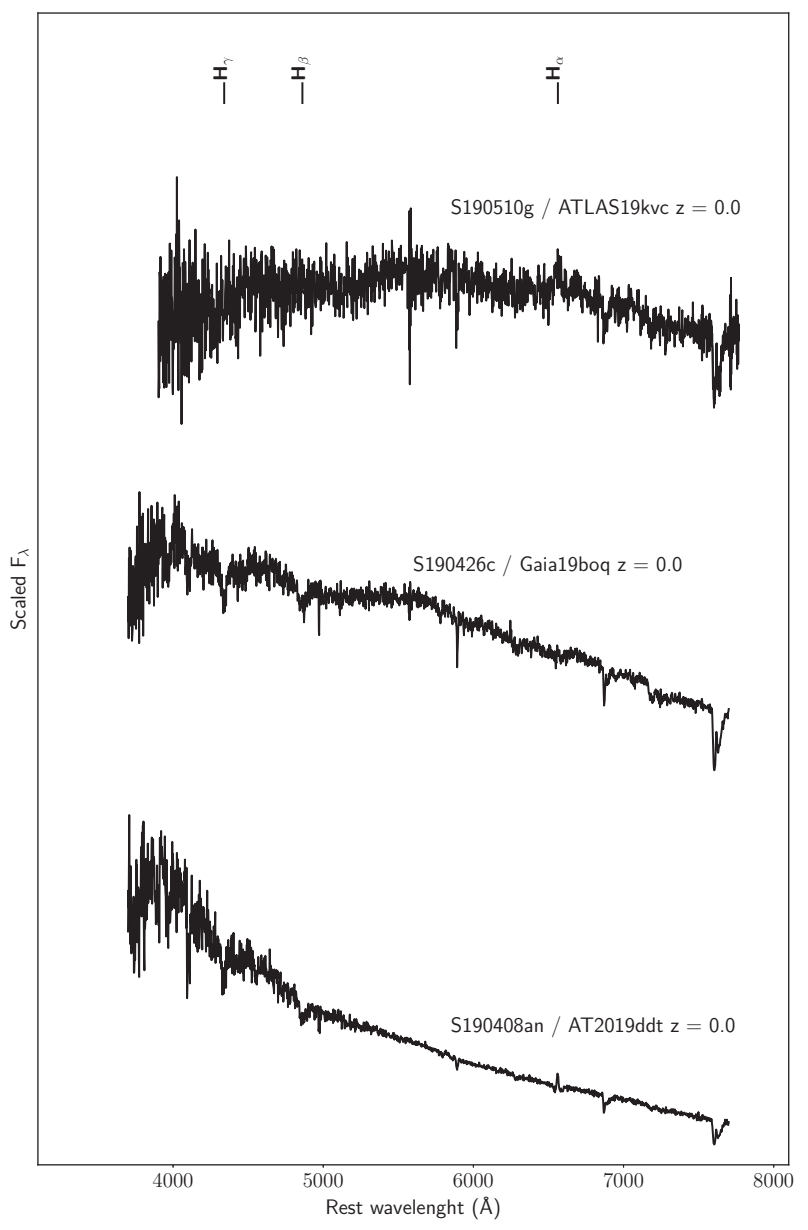

Fig. 1. Spectra of cataclismic variables. S190426c / Gaia19boq shows $\mathrm{H} \alpha$ with PCyg profile and other Balmer series in absorption. Emission $\mathrm{H} \alpha$ and absorption $\mathrm{H} \beta, \mathrm{H} \gamma$ for AT 2019ddt is representated. ATLAS19kvc revealed both $\mathrm{H} \alpha$ and $\mathrm{H} \beta$ in emission.

AT 2019ddt/MASTER OT J231043.63 $+\mathbf{4 7 0 9 5 6 . 4}$ was discovered by MASTER robotic telescopes eight hours after the GW event as the 16.5 transient (filterless) (Lipunov 2019). The spectrum obtained by GTC 10.4-m (Valeev 2019a) shows the zero-redhifted emission $\mathrm{H} \alpha$ (Fig. 1). Both $\mathrm{H} \beta$ and $\mathrm{H} \gamma$ were in absorption.

\section{S190425Z}

The S190425z (Ligo Scientific Collaboration \& VIRGO Collaboration 2019a) event was detected by two detectors (L1, V1) starting at 2019-04-25 08:18:25 UTC and was classified as binary neutron star merger with high propability $(>99 \%)$. The distance estimation is $\mathrm{d}=156 \pm 41 \mathrm{Mpc}$ and the error box $1378 / 7461 \mathrm{deg}^{2}$ for $50 / 90 \%$ confidence.

AT 2019dzw/ZTF19aarzaod The candidate was identified with ZTF (Zwicky Transient Facil- 
ity) group $\sim 2$ hours after the GW event close to the nearby host galaxy at photometrical redshift 0.028 (Kasliwal 2019). Our 3700-7500 ̊ spectrum reveals the $\mathrm{SN}$ features at $\mathrm{z}=0.0279 \pm 0.0005$ (Castro-Tirado 2019a) in agreement with classifications which made by other groups.

\section{5. $\mathrm{S} 190426 \mathrm{C}$}

On Apr, 26 at 15:22:17 UTC three GW detectors (H1, L1, V1) discovered the S190426c (Ligo Scientific Collaboration \& VIRGO Collaboration 2019b) event, firstly classified as binary neutron stars merging with $49 \%$ likehood. The distance was estimated as $423 \pm 128 \mathrm{Mpc}$ and sky localisation $472 / 1932 \mathrm{deg}^{2}$ for $50 / 90 \%$ confidence, refined to $214 / 1131 \mathrm{deg}^{2}$ errorbox. 3 month later it was reclassificated as having probability $58 \%$ of being non-astrophysical in origin. Dozen of the candidates were announced in the Andreoni (2019b); Perley (2019)

DG19kplb This candidate was discovered in DECam data (Andreoni 2019b). Using GTC spectra DG19kplb was classified as broad-line past maximum SN Ic at the host galaxy redshift $\mathrm{z}=0.0912 \pm 0.0005$ (Valeev 2019b).

DG19ytre blue transient with $\mathrm{r}=20.69$ and $\mathrm{z}$ $=21.29$ was announced by Andreoni (2019b). On GTC/OSIRIS blue part spectrum it resembles the SN Ia (similar to SN 2011fe Tsvetkov (2013)) at the host galaxy redshift $\mathrm{z}=0.1386 \pm 0.0005$ (Valeev 2019b).

AT 2019snh/ZTF19aaslzjf was discovered as the $\mathrm{g}=21.09$ and $\mathrm{r}=20.54$ transient close to the host galaxy with unknown redshift (Perley 2019). Both reshifts $\mathrm{z}=0.086 \pm 0.005$ (Fig 2) from the SN Ia like spectrum and from the emission lines of the galaxy coincide (Hu 2019a).

ZTF19aasmddt transient with $\mathrm{g}=20.00$ was announced by ZTF group (Perley 2019). The photometrical redshift of its host galaxy was known with the high uncertainty $0.08 \pm 0.04$. Our spectroscopy (Hu 2019a) refined the redshift to $\mathrm{z}=0.028 \pm 0.005$ and transient classified as SNII before maximum (Fig 3).

AT 2019sne/ZTF19aasmftm was found by ZTF group $\sim 18^{h}$ after GW event Perley (2019). Few days before maximum SN Ia spectrum at host galaxy with $\mathrm{z}=0.156 \pm 0.005$ supports the rising magnitude reported in Perley (2019).

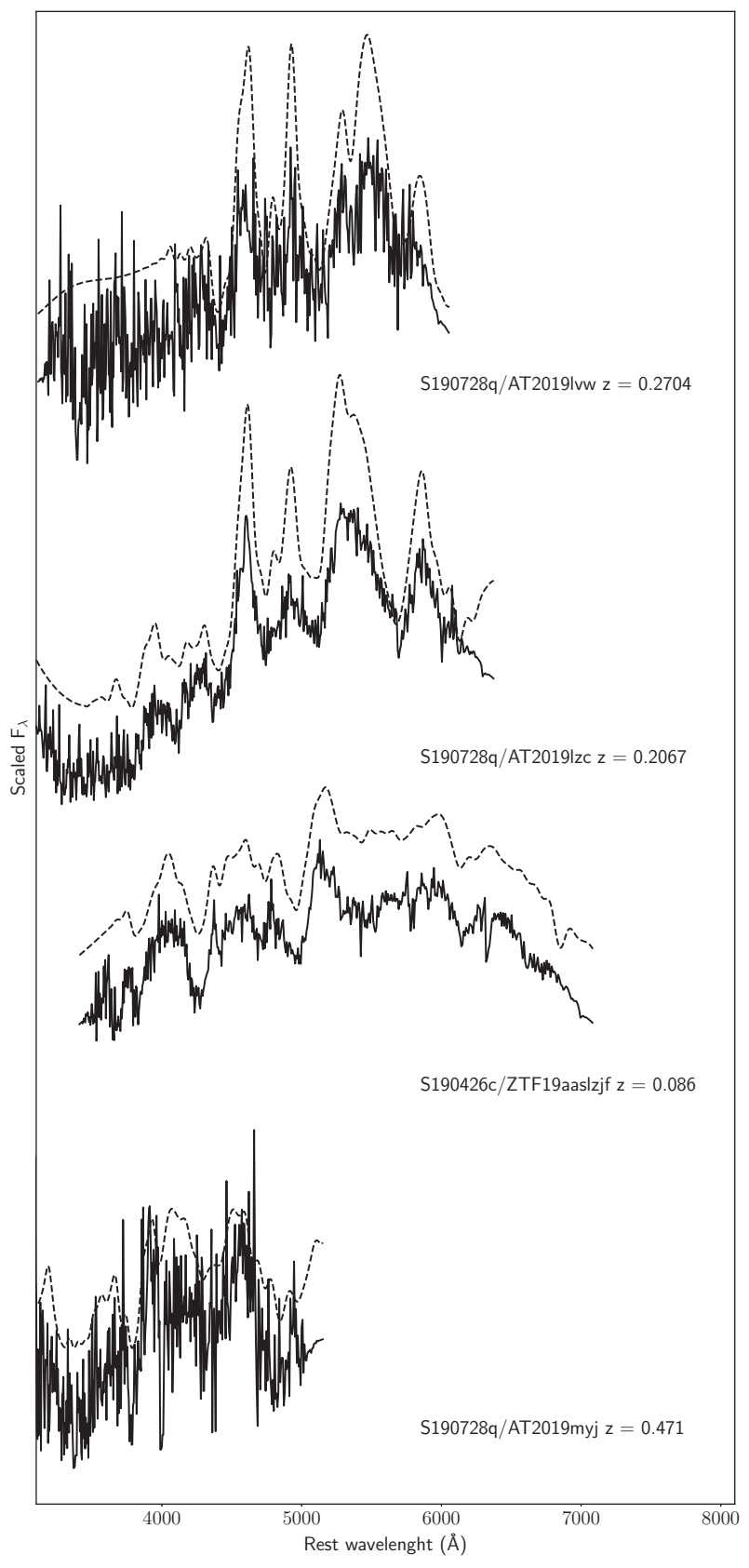

Fig. 2. Spectra of SN Ia: S190728q / AT 2019myj at z $=$ 0.471, S190426c / ZTF19aaslzjf at $\mathrm{z}=0.086$, S190728q / AT 2019lzc at $\mathrm{z}=0.2067$ and S190728q / AT 2019lvw at $\mathrm{z}=0.2704$ are presented. Dashed lines show the template spectra. Small shifts for better representation are applied.

ZTF19aassfws coincide with the center of the host galaxy and the simultaneous observations by PanStarrs did not confirm this faint source (Perley 2019). The spectrum is fitted by absorption galaxy template and there was not any variation in spectrum in 15-day interval. 


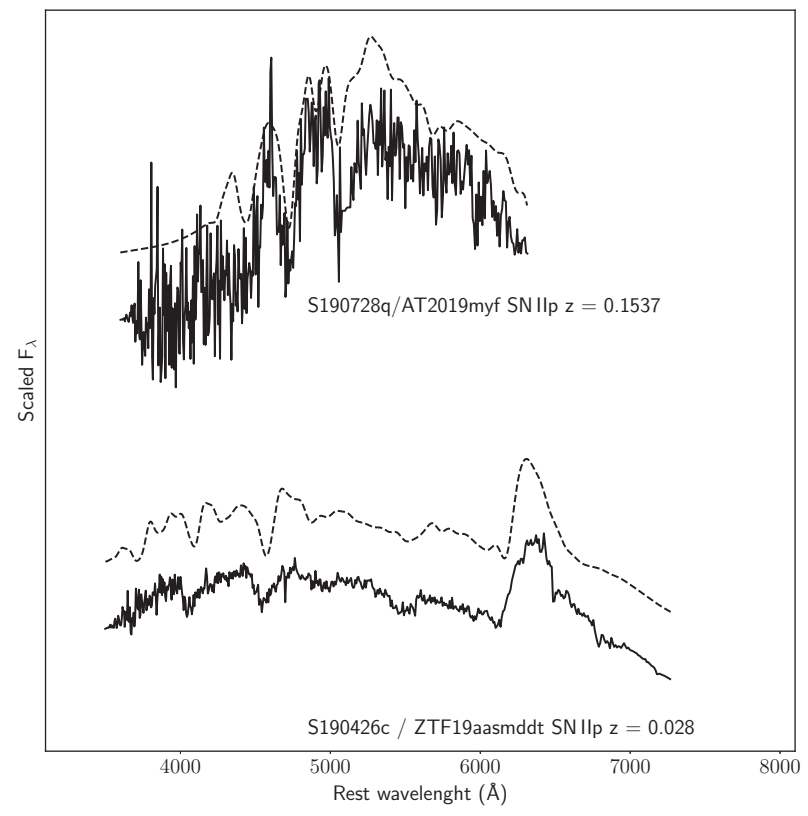

Fig. 3. Spectra of SN II. Dashed lines show the template spectra. Small shifts for better representation are applied.

ZTF19aaslszp was discovered on Apr 27 with g $=20.84$ (Perley 2019). However, on May 1 it was not visible on the image up to the $23 . \mathrm{m}$.

2019egk/Gaia19boq was announced by Gaia Alerts team (Kostrzewa-Rutkowska 2019). The historical observations show the transient clearly detectable before the GW event. $\mathrm{H} \alpha$ shows the $\mathrm{P}$ Cyg profile and other Balmer series were in absorption. We concluded that this was cataclismic variable in Galaxy (Sanchez-Ramirez 2019).

\section{S190510G}

Binary neutron stars merging system was registred by three Ligo/Virgo detectors (H1, L1, V1) on 2019-05-10 03:00:00 UT (Ligo Scientific Collaboration \& VIRGO Collaboration 2019c). The propability of non terrestial signal was only $2 \%$. Despite the small localization area, the GW analysis places S190510g at the fairly large distance of $269 \pm 108 \mathrm{Mpc}$. The 50/90\% confidence errorboxes were only $31 / 1166 \mathrm{deg}^{2}$. After the reanalysing of Ligo/Virgo data the mostly terrestial origin of signal ( $58 \%$ propability) and only $42 \%$ chance of BNS was reported.

AT 2019far/PS19aaf was found inside the GW errorbox $\sim 8$ hours after event (Srivastav 2019). Follow-up spectroscopy (Castro-Tirado 2019b) revealed the SN IIp features for the transient and redshift $\mathrm{z}=0.0583 \pm 0.0005$ for the host galaxy.
AT 2019ezb/PS19xv was detected on 2019-0510 07:12:00 UT (Srivastav 2019). Due to the weather we could exposure the optical spectrum only on May 14. The transient was classified as SN Ic and host galaxy redshift measurement pointed out $\mathrm{z}=0.0712$ \pm 0.0005 (Castro-Tirado 2019b).

AT 2019fsk/ATLAS19kvc was announced only on May 21 by Atlas team. However, we obtained the spectrum of the transient and classify it as a cataclismic variable in Galaxy with $\mathrm{H} \alpha$ and $\mathrm{H} \beta$ emissions.

\section{S190728Q}

The most accurate sky localisation was announced for S190728q event (Ligo Scientific Collaboration \& VIRGO Collaboration 2019d) after the offline reanalysing of the records from all three $\mathrm{GW}$ detectors (H1, L1, V1). The 50/90\% confidence errorboxes were only $24 / 104 \mathrm{deg}^{2}$ at $874 \pm 171 \mathrm{Mpc}$ distance. After the data were reanalysed the signal is mostly associated with the binary system in mass gap or binary black holes one.

The optical transients were announced in GCN telegrams (Goldstein 2019a; Huber 2019; SoaresSantos 2019a).

AT 2019lzb/PS19dxc was discovered by PanSTARRS team (Huber 2019). The optical spectroscopy uncover the AGN nature of the galaxy center (Fig 4). The redhift $\mathrm{z}=0.019 \pm 0.001$ was estimated using the narrow galactic emissions (Valeev 2019c).

AT 2019lzc/PS19dxb was firstly observed by Pan-STARRS $\sim 3.5$ hours after the GW event $(\mathrm{Hu}-$ ber 2019). The narrow emission lines of host galaxy hinted the redshift $\mathrm{z}=0.207 \pm 0.005$. The broad emissions spectrum was fitted by SN Ia template.

AT 2019lzt/PS19dxn transient with fast rising brightness was announced by Huber (2019). Followup spectroscopy allowed us (Castro-Tirado 2019c) to estimate the redshift $\mathrm{z}=0.193 \pm 0.001$ and define the type of supernova - SN Ia.

AT 2019lzg/PS19dxf was announced by Huber (2019). We obtained the 1.2 exposure spectrum of this target. Zero-redshifted $\mathrm{H} \alpha$ is clearly detected. The best fit with M6V template is shown in Fig. 5. Our deep photometry gives $\mathrm{g}=27.3 \pm 0.8, \mathrm{r}=25.7$ $\pm 0.3, \mathrm{i}=23.83 \pm 0.15, \mathrm{z}=22.35 \pm 0.05$. LePhare spectral energy distribution fit also reveals the M6V solution. 


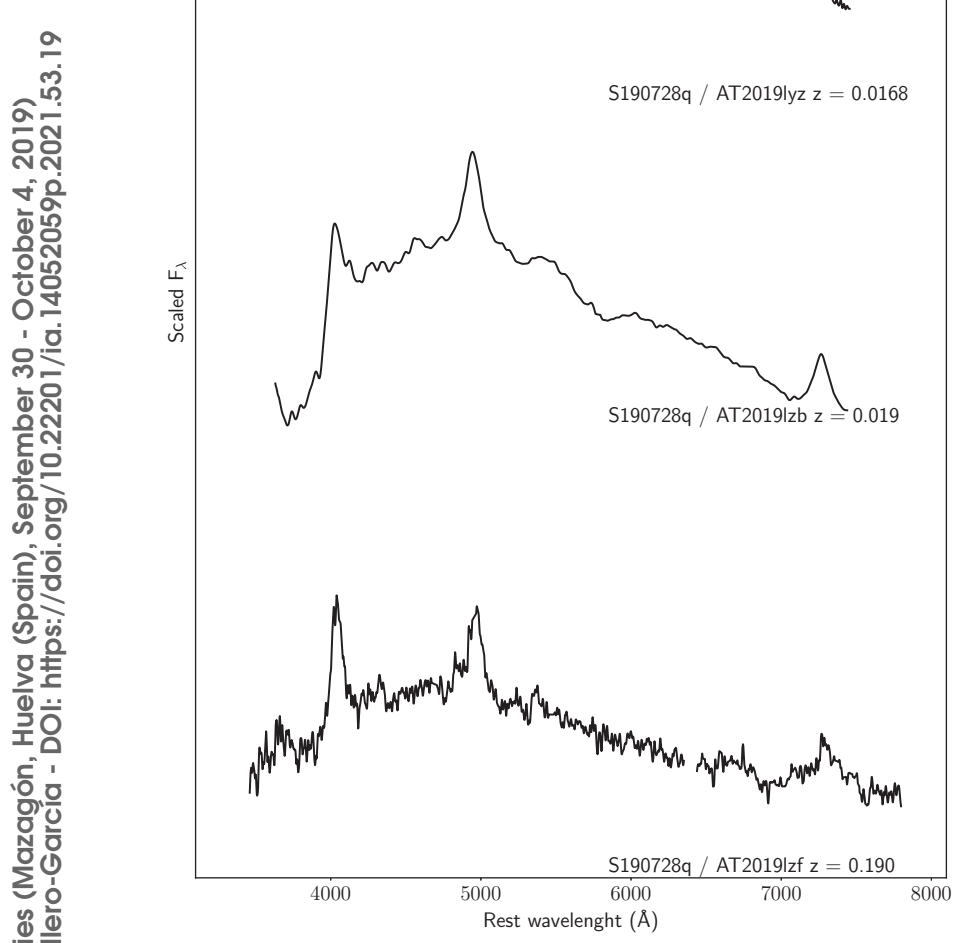

Fig. 4. Spectra of AGNs S190728q / AT2019lzf, S190728q / AT2019lzb, S190728q / AT2019lyz are presented below.

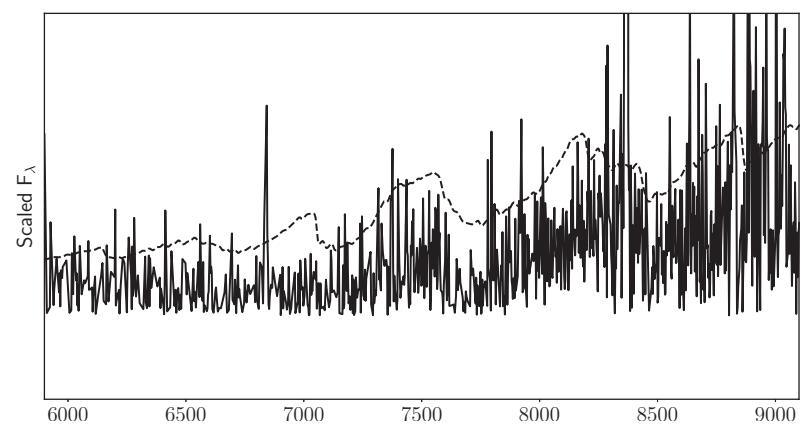

Fig. 5. Spectrum of flaring M-dwarf AT 2019lzg. Template of M6V (shifted) is shown by dashed line. Result of 1.2 exposure for $\mathrm{r}=25.7 \pm 0.3$ star.

AT 2019lzf/PS19dxg was an activity of galactic nucleus. The spectrum was very similar to that of AT 2019lzb (see the Fig. 4 ). However, only the presence of the third broad feature in the R1000R spectrum hinted this similarity. The matching of both spectra revealed the redshift $0.190 \pm 0.001$.

AT 2019lyz/PS19dwz was detected on 2019-0728 12:43:12 UT close to the center of nearby galaxy (Huber 2019). Our spectrum (Fig. 4) showed the broad emission and hinted the nucleus activity at redshift $\mathrm{z}=0.0168 \pm 0.0005$.

AT 2019myi/desgw-190728e located in 2 arcsec from the center of the host galaxy (Soares-Santos 2019a). It was discovered $\sim 21$ hours after the GW event with $\mathrm{r}=21^{\mathrm{m}} 92$. The analysis of the blue spectrum shows the galactic emissions at redshift $\mathrm{z}=$ $0.1827 \pm 0.0005$. Template fitting procedure indicates that the spectrum most resembles SN Ia after maximum.

AT 2019myh/desgw-190728d position in the sky is close to the center of the diffuse galaxy on PanStarrs images. It was announced in SoaresSantos (2019a) with $\mathrm{r}=22 \mathrm{~m} 29$. The redshift $\mathrm{z}=$ $0.2909 \pm 0.0005$ measured from the galactic emissions is confirmed by template fitting output. The transient was classified as SN Ia after maximum.

AT 2019myg/desgw-190728c brightness rised slowly when the object was detected by DESGW team (Soares-Santos 2019a). Although we do not see the galaxy in direct images, its emissions are clearly uncovered in our spectrum. Our photometrical measurement gives $\mathrm{r}=22^{\mathrm{m}} \cdot 55 \pm 0.05$ at 2019-08-11 03:46 UT. Spectrum with broad features corresponds to SN Ia at $\mathrm{z}=0.0122 \pm 0.0005$.

AT 2019myf/desgw-190728b was found in outskirts ( 3.6 arcsec away) of the host galaxy (SoaresSantos 2019a). We took 900 seconds exposure spectrum, covering both the galaxy and the transient positions. The galaxy redshift $\mathrm{z}=0.1537 \pm 0.0005$ confirmed the transient redshift from the fitting of its spectrum with SN 2006bp (SN IIp) after maximum template.

AT 2019myj/desgw-190728a was discovered by DESGW team on 2019-07-29 03:05:45 UT (SoaresSantos 2019a). The fading 0m 25 / 3 days was noted. The galaxy was found at 4 arcsec in north direction, but the spectroscopy reveals the redshift of this galaxy $\mathrm{z}=0.5940 \pm 0.0005$, which is not consistent with solution for transient $\mathrm{z}=0.471 \pm 0.004$. The spectrum was good fitted with SN Ia template.

AT 2019lvw/DG19arsob (Goldstein 2019a) located in 4.5 arcsec from two galaxies. The disk of one galaxy is barely visible up to the position of the 
transient. Our analysis (Hu 2019b) indicated that the optical spectrum of target matched to the SN Ia template at $\mathrm{z}=0.2704 \pm 0.0005$.

\section{S190814BV}

The LIGO/Virgo Collaborations detected the GW event S190814bv on 2019-08-14 21:10:39 UT (Ligo Scientific Collaboration \& VIRGO Collaboration 2019e). Pipeline analysing of the records from all three GW detectors (H1, L1, V1) were used. The false alarm rate of the event was approximately one in $10^{25}$ years. After revision based on new parameter estimation the event was classified as "black hole - neutron star merger" with $99 \%$ probability. Offline analysis improved the localisation errorbox to $5 / 23 \mathrm{deg}^{2}$ (50/90\% confidence) at $267 \pm 52 \mathrm{Mpc}$ distance. Andreoni (2019a) investigated the limit of the optical/near-infrared counterpart for this event.

AT 2019nqq/desgw-190814c was announced by DESGW team (Herner 2019b). SN IIP post maximum template (Lopez-Cruz 2019b) best matched to the spectrum of transient obtained on Aug, 20. Template matching algorithm and galactic narrow emission lines revealed redshift $\mathrm{z}=0.071 \pm 0.001$.

AT 2019nqz/DG19ayfjc was detected close to center of the host galaxy (Goldstein 2019b). The upper limit magnitude for the transient was $\mathrm{i}=23 \mathrm{~m}^{\mathrm{m}} 4$ on stacked $3 \times 10$ seconds image. However, we measured the redshift $\mathrm{z}=0.1076 \pm 0.0005$ of host galaxy (Lopez-Cruz 2019a) and concluded that it is outside the expected LVC redshift range.

AT 2019nqc/DG19aqbkc with $i=20.6$ found on 2019-08-16 UT 08:24 by DECam-GROWTH team (Andreoni 2019c). Our spectroscopy (Lopez-Cruz 2019a) reveals narrow galaxy lines at redshift 0.078 \pm 0.001 . Cross-correlating the transient spectrum (broad lines) with supernova template spectra in SNID, we found a good match to the spectra of SNIIP at about week after maximum at same redshift in agreement with the result reported by Buckley (2019)

AT 2019obc/desgw-190814q was found in DES J005815.91-240823.4 galaxy with photometric redshift $0.28 \pm 0.18$ (Soares-Santos 2019d). We observed the transient 6 days after detection (CastroTirado 2019d). The redshift, as derived from SNID, is $\mathrm{z}=0.216$, linking the transient to the host galaxy $(\mathrm{z}=0.2161 \pm 0.0005$ derived from the galactic emission line $[\mathrm{O} \mathrm{III}])$.
AT 2019nxe/desgw-190814j was announced by DESGW group. It is located in outskirts of the host galaxy (Soares-Santos 2019c) with photometric redshift $\mathrm{z}=0.21 \pm 0.21$. However our spectroscopy reveals the redshift $0.0777 \pm 0.0005$, according to the narrow emission lines of $\mathrm{H} \alpha$, [N II], [S II]. On Aug 23 02:15 UT the spectrum of SN Ia was exposured for $\mathrm{r}=20.39 \pm 0.07$ target (Castro-Tirado 2019d).

AT 2019nzr/desgw-190814m coincided with the center of the host galaxy (Soares-Santos 2019c). The photometry for transient $\mathrm{i}=23.78$ (discovery magnitude) and for host galaxy $\mathrm{i}=18.45$. Its red spectrum was obtained on 2019-08-23 03:00 UT. The measured redshift of the host galaxy $\mathrm{z}=0.2589$ \pm 0.0005 coincides with the other galaxy $\sim 7 \operatorname{arcsec}$ away in NE direction. We could not classify the spectrum, but it did not fit with AGN template.

AT 2019nte/desgw-190814f was detected (Herner 2019a) near the galaxy GALEXASC J013413.83-314318.3 with photometric redshift 0.07 . We did not found the transient $0.5 \operatorname{arcsec}$ (or 2 pixels) away from the center of galaxy. Our spectroscopy revealed the redshift $0.0698 \pm 0.0005$, this result is consistent with that reported in Soares-Santos (2019e).

AT 2019odc/desgw-190814r was discovered $\sim 0.7$ arcsec away from center of host $\mathrm{SBb}$ galaxy on 2019-08-17 04:52:19 UT with i $=21.57$ (SoaresSantos 2019b). Unfortunately, due to the weather we observed transient position only 13 days later. We could not detect the transient itself. Using galactic emissions we measured the redhift of galaxy $\mathrm{z}=0.0540 \pm 0.0005$ (Hu 2019c).

AT 2019omt/desgw-190814v was announced by DESGW team Soares-Santos (2019b). Spectroscopy was performed two weeks later. The galaxy redhift $\mathrm{z}=0.1564 \pm 0.0005$ is outside of the expected LVC redshift range. Our transient spectrum resembled a post-maximum SN IIL one.

Acknowledgments: The work is based on observations made with the 10.4-m Gran Telescopio de Canarias. VAF was supported by RFBR 18-2921030 grant. JAF acknowledges financial support provided by the Spanish Agencia Estatal de Investigación (grant PGC2018-095984-B-I00), by the Generalitat Valenciana (PROMETEO/2019/071) and by the European Union's Horizon 2020 research and innovation (RISE) programme H2020-MSCA-RISE2017 (Grant No. FunFiCO-777740). 
TABLE 1

CLASSIFICATION OF TRANSIENTS. WE LIMIT OUR TARGET LIST TO THE CANDIDATES OBSERVED PRIOR 2019 SEP, 1

\begin{tabular}{|c|c|c|c|c|}
\hline GW event & Name & RA & DEC & classification \\
\hline S190408an & AT 2019ddt /MASTER OT & $23: 10: 43.63$ & $47: 09: 56.4$ & $\mathrm{CV}$ at $\mathrm{z}=0$. \\
\hline S190425z & ZTF19aarzaod & $17: 31: 09.957$ & $-08: 27: 02.60$ & SNIIn $\mathrm{z}=0.0279 \pm 0.0005$ \\
\hline S190426c & DG19ytre & $11: 11: 02.48$ & $00: 31: 37.8$ & $\mathrm{SN} \mathrm{Ia} \mathrm{z}=0.1386 \pm 0.0005$ \\
\hline S190426c & DG19pklb & 11:14:38.06 & $-06: 58: 31.0$ & SN Ic $\mathrm{z}=0.0912 \pm 0.0005$ \\
\hline S190426c & ZTF19aasmddt & $19: 57: 00.13$ & 09:42:06.1 & $\mathrm{SNII} z=0.028 \pm 0.005$ \\
\hline S190426c & ZTF19aassfws & $19: 54: 40.28$ & 61:14:00.0 & galaxy spectrum \\
\hline S190426c & ZTF19aaslszp & $20: 05: 22.4$ & $53: 20: 36.6$ & not visible \\
\hline S190426c & ZTF19aaslzjf & $21: 22: 30.31$ & $65: 48: 48.6$ & SN Ia $z=0.086 \pm 0.005$ \\
\hline S190426c & ZTF19aasmftm & $21: 43: 36.11$ & $77: 49: 53.4$ & SN Ia $z=0.156 \pm 0.005$ \\
\hline S190426c & AT 2019egk/Gaia19boq & $21: 16: 23.41$ & $58: 53: 27.2$ & $\mathrm{CV}$ at $\mathrm{z}=0$. \\
\hline $\mathrm{S} 190510 \mathrm{~g}$ & AT 2019far & $14: 17: 22.53$ & 05:01:08.7 & SN IIp $z=0.0583 \pm 0.0005$ \\
\hline S190510g & AT $2019 \mathrm{ezb}$ & $13: 43: 59.78$ & $01: 50: 32.7$ & SN Ic $\mathrm{z}=0.0712 \pm 0.0005$ \\
\hline S190510g & ATLAS19kvc & $17: 19: 41.85$ & 01:04:45.1 & $\mathrm{CV}$ at $\mathrm{z}=0$. \\
\hline S190728q & AT 2019lzb & $20: 45: 55.34$ & 02:05:53.5 & $\mathrm{AGN} \mathrm{z}=0.019 \pm 0.002$ \\
\hline S190728q & AT 2019lzc & $20: 52: 54.7$ & $02: 31: 43.6$ & SN Ia $\mathrm{z}=0.207 \pm 0.005$ \\
\hline S190728q & AT 2019lzt & $21: 01: 13.26$ & $13: 29: 39.0$ & $\mathrm{SN} \mathrm{Ia} \mathrm{z}=0.193 \pm 0.001$ \\
\hline S190728q & AT 2019lzg & $21: 03: 16.16$ & $14: 22: 50.9$ & M-dwarf $\mathrm{z}=0$. \\
\hline S190728q & AT 2019lzf & 21:11:56.20 & $17: 14: 27.2$ & $\mathrm{AGN} z=0.190 \pm 0.001$ \\
\hline S190728q & AT 2019lyz & 21:14:09.78 & $13: 37: 04.8$ & $\mathrm{AGN} \mathrm{z}=0.0168 \pm 0.0005$ \\
\hline S190728q & AT 2019lvw & $20: 54: 35.1$ & $07: 34: 00.3$ & $\mathrm{SN} \mathrm{Ia} \mathrm{z}=0.2704 \pm 0.0005$ \\
\hline S190728q & AT 2019myf & $21: 15: 19.60$ & $17: 36: 20.1$ & SN IIp $\mathrm{z}=0.1537 \pm 0.0005$ \\
\hline S190728q & AT 2019myg & $20: 59: 12.90$ & $08: 33: 35.6$ & $\mathrm{SN} \mathrm{Ia} \mathrm{z}=0.0122 \pm 0.0005$ \\
\hline S190728q & AT 2019myh & $21: 09: 36.80$ & $12: 07: 30.6$ & $\mathrm{SN} \mathrm{Ia} \mathrm{z}=0.2909 \pm 0.0005$ \\
\hline S190728q & AT 2019myi & $21: 05: 31.70$ & $12: 23: 49.7$ & $\mathrm{SN} \mathrm{Ia} \mathrm{z}=0.1827 \pm 0.0005$ \\
\hline S190728q & AT 2019myj & 21:07:29.10 & $13: 15: 01.7$ & $\mathrm{SN} \mathrm{Ia} \mathrm{z}=0.471 \pm 0.004$ \\
\hline S190814bv & AT 2019nqq & $01: 23: 49.22$ & $-33: 02: 05.0$ & SN IIP $\mathrm{z}=0.071 \pm 0.001$ \\
\hline S190814bv & AT 2019nqz & $00: 46: 46.42$ & $-24: 20: 12.1$ & no object, $\mathrm{z}=0.1076 \pm 0.0005$ \\
\hline S190814bv & AT 2019nqc & 01:29:03.67 & $-32: 42: 18.5$ & SN IIP $\mathrm{z}=0.078 \pm 0.001$ \\
\hline S190814bv & desgw-190814q & 00:58:16.02 & $-24: 08: 23.2$ & $\mathrm{SN} \mathrm{Ia} \mathrm{z}=0.2161 \pm 0.0005$ \\
\hline S190814bv & AT 2019nxe & $00: 46: 16.81$ & $-24: 22: 21.2$ & $\mathrm{SN} \mathrm{Ia} \mathrm{z}=0.0777 \pm 0.0005$ \\
\hline S190814bv & AT 2019nzr & $00: 47: 21.41$ & $-24: 34: 36.6$ & unknown, $\mathrm{z}=0.2589 \pm 0.0005$ \\
\hline S190814bv & AT 2019nte & $01: 34: 13.77$ & $-31: 43: 18.1$ & no object, $\mathrm{z}_{\text {galaxy }}=0.0698 \pm 0.0005$ \\
\hline S190814bv & AT 2019odc & 00:46:01.68 & $-25: 27: 33.1$ & no object, $\mathrm{z}_{\text {galaxy }}=0.0540 \pm 0.0005$ \\
\hline S190814bv & AT 2019omt & $00: 59: 26.73$ & $-25: 59: 41.3$ & SN IIL $z=0.1564 \pm 0.0005$ \\
\hline
\end{tabular}

\section{REFERENCES}

Abbott, B. P. 2017, ApJ, 848, L12

Abbott, B. P. \& LIGO \& Virgo Scientific Collaboration. 2016, PhRvL, 116, 061102 2017, PhRvL, 118, 221101

Andreoni, I. et al., 2019a, arXiv e-prints, arXiv:1910.13409 2019b, GCN, 24268, 1 2019c, GCN, 25362, 1

Blondin, S. \& Tonry, J. L. 2007, ApJ, 666, 1024

Buckley, D. 2019, GCN, 25481, 1

Castro-Tirado, A. J. et al., 2019a, GCN, 24214, 1 . 2019b, GCN, 24535, 1 2019c, GCN, 25253, 1 
2019d, GCN, 25543, 1

Cepa, J., Aguiar, M., Escalera, V. G., et al. 2000, SPIE, 4008, 623

Gehrels, N., Cannizzo, J. K., Kanner, J., Kasliwal, M. M., Nissanke, S., \& Singer, L. P. 2016, ApJ, 820, 136

Goldstein, D. et al., 2019a, GCN, 25215, 1 2019b, GCN, 25391, 1

Herner, K. 2019a, GCN, 25398, 1 2019b, GCN, 25373, 1

$\mathrm{Hu}$, Y. D. et al, 2019a, GCN, 24359, 1 2019b, GCN, 25276, 1 2019c, GCN, 25588, 1

Huber, M. 2019, GCN, 25224, 1

Kasliwal, M. M. 2019, GCN, 24191, 1

Kostrzewa-Rutkowska, Z. 2019, GCN, 24344, 1

Ligo Scientific Collaboration \& VIRGO Collaboration. 2019a, GCN, 24168, 1 2019b, GCN, 24237, 1 2019c, GCN, 24442, 1 2019d, GCN, 25187, 1 2019e, GCN, 25324, 1

Lipunov, V. 2019, GCN, 24084, 1

Lopez-Cruz, O. et al., 2019a, GCN, 25571, 1 2019b, GCN, 25419, 1

Perley, D. A. et al., 2019, GCN, 24331, 1

Sanchez-Ramirez, R. et al., 2019, GCN, 24368, 1

Soares-Santos, M. et al., 2019a, GCN, 25302, 1 2019b, GCN, 25486, 1 $2019 \mathrm{c}, \mathrm{GCN}, 25425,1$ 2019d, GCN, 25438, 1 2019e, GCN, 25784, 1

Srivastav, S. 2019, GCN, 24517, 1

Tsvetkov, D. Y., Shugarov, S. Y., Volkov, I. M., et al. 2013, CoSka, 43, 94

Valeev, A. F. et al., 2019a, GCN, 24092, 1 2019b, GCN, 24317, 1 2019c, GCN, 25261, 1 